\title{
In Silico Approach for Immunohistochemical Evaluation of a Cytoplasmic Marker in Breast Cancer
}

\author{
Claudia Mazo ${ }^{1,2,3, *,+}(\mathbb{1})$, Estibaliz Orue-Etxebarria ${ }^{4, \ddagger}$, Ignacio Zabalza ${ }^{5, \ddagger}$, Maria d. M. Vivanco ${ }^{4, \ddagger}$, \\ Robert M. Kypta ${ }^{4,6, \ddagger}$ (1) and Andoni Beristain $1, \mp$ \\ 1 Vicomtech, eHealth and Biomedical Applications, 20009 San Sebastian-Donostia, Spain; \\ aberistain@vicomtech.org \\ 2 School of Computer Science, University College Dublin, D14 YH57 Dublin, Ireland \\ 3 CeADAR: Centre for Applied Data Analytics Research, D04 V1 W8 Dublin, Ireland \\ 4 CIC bioGUNE, Center for Cooperative Research in Biosciences, 48160 Bilbao, Spain; \\ eorue@cicbiogune.es (E.O.-E.); mdmvivanco@cicbiogune.es (M.d.M.V.); rkypta@cicbiogune.es (R.M.K.) \\ 5 Department of Pathology, Galdakao-Usansolo Hospital, 48960 Galdakao, Spain; \\ ignacio.zabalzaestevez@osakidetza.eus \\ 6 Imperial College London, Department of Surgery and Cancer, London SW7 2AZ, UK \\ * Correspondence: claudia.mazovargas@ucd.ie \\ + Current address: Affiliation 2 and 3. \\ $\ddagger$ Authors with unequally contribution to this work.
}

Received: 23 November 2018; Accepted: 12 December 2018; Published: 15 December 2018

\begin{abstract}
Breast cancer is the most frequently diagnosed cancer in women and the second most common cancer overall, with nearly 1.7 million new cases worldwide every year. Breast cancer patients need accurate tools for early diagnosis and to improve treatment. Biomarkers are increasingly used to describe and evaluate tumours for prognosis, to facilitate and predict response to therapy and to evaluate residual tumor, post-treatment. Here, we evaluate different methods to separate Diaminobenzidine (DAB) from Hematoxylin and Eosin (H\&E) staining for Wnt-1, a potential cytoplasmic breast cancer biomarker. A method comprising clustering and Color deconvolution allowed us to recognize and quantify Wnt-1 levels accurately at pixel levels. Experimental validation was conducted using a set of 12,288 blocks of $m \times n$ pixels without overlap, extracted from a Tissue Microarray (TMA) composed of 192 tissue cores. Intraclass Correlations (ICC) among evaluators of the data of $0.634,0.791,0.551$ and 0.63 for each Allred class and an average ICC of 0.752 among evaluators and automatic classification were obtained. Furthermore, this method received an average rating of 4.26 out of 5 in the Wnt- 1 segmentation process from the evaluators.
\end{abstract}

Keywords: breast cancer; Wnt-1; immunohistochemistry (IHC); automatic segmentation; automatic quantification

\section{Introduction}

Breast cancer is one of the most common types of cancer in most European and American countries and the second leading cause of cancer death [1-3]. About 266,120 new cases of invasive breast cancer are diagnosed in women in Europe every year. Breast cancer represents almost a sixth (16\%) of all cases of cancer in males and females combined. However, mortality rates have decreased as a result of earlier diagnosis and improved therapies, according to the American Cancer Society and International Agency of Research Cancer [4]. The WNT genes encode a family of 19 secreted short-range signalling proteins involved in the regulation of cell fate, proliferation, migration, polarity and death, processes that play important roles in cancer initiation and/or progression [5]. Increased expression of the Cancer Stem Cell (CSC) marker SOX2 in human breast cancer activates Wnt-1 signaling to promote resistance to 
tamoxifen, the most common therapy for Estrogen Receptor-positive (ER+) breast cancer [6]. There are nineteen Wnt-1 family members, several of which may play a role in breast cancer. Among them is Wnt-1, which was originally named int-1 because integration of the Mouse Mammary Tumor Virus (MMTV) into its gene locus induces mammary tumors in mice [7]. Consistent with this, Wnt-1 increases the CSC population [8] and shRNA-mediated Wnt-11 silencing in a metastatic mouse breast cancer cell line reduces expression of CSC markers and tumor formation [9]. There are fewer studies of Wnt-1 in human breast cancer. However, Wnt-1 protein has been detected in tumors of Korean patients with invasive ductal carcinoma [10] and has been reported to be upregulated in human breast tumors, compared with adjacent normal tissue [11]. Together, these data suggest that Wnt-1 could be a useful biomarker and therapeutic target in breast cancer. In addition, Wnt-1 expression may be an indicator of poor prognosis in cutaneous squamous cell carcinoma [12] and in non-small cell lung cancer [13], and a potential biomarker of recurrence in Hepatitis C-related hepatocellular carcinoma [14]. Although Wnt-1 is a secreted protein, Wnt-1 in the extracellular space is undetectable by immunohistochemistry, as is the case for most secreted proteins, which are more readily detected in the cytoplasm, being enriched in organelles of the secretory pathway. In this study, we therefore used Wnt-1 as an example of a cytoplasmic marker to test our in silico method.

The classification and quantification of biomarker expression levels is carried out by pathologists, biologists and related professionals on a limited region of a tumor. Manual scoring is normally carried out by at least two scientists with experience in scoring the antigen, and at least including one pathologist. In this study, immunoreactivity for Wnt-1 was scored using a semi-quantitative evaluation based on the Allred system [15]. An in silico approach may help to improve the reproducibility of scoring among pathologists, which is common according to the subjectivity of pathological diagnosis. It may also increase the number of cases that an expert can analyze. Thus, automated scoring will improve patient quality of life and reduce associated health-care costs. Additionally, this type of approach may be being helpful for the purpose of medical education [16]. Furthermore, an in silico approach could provide support to methods for discovering new biomarkers and mitigating issues related to inter-observer variability (e.g., bias, time, difficulty, costs, and impracticality) [17].

Some in silico approaches can recognize biomarkers-e.g., Estrogen Receptor (ER), Progesterone Receptor (PR), Phosphatase and Tensin Homolog (PTEN), Human Epidermal Growth Factor Receptor 2 (HER2), and Ki-67-in histopathology images [18-22]. A free ImageJ plugin and Internet-based web application, called ImmunoRatio, for automatic segmentation and quantification of ER, PR, and Ki-67 immunohistochemistry (IHC) in breast cancer tissue sections is presented in [18]. Color deconvolution, adaptive thresholding and median filter are used in ImmunoRatio. This approach achieved a correlation coefficient of 0.98 . An important aspect to take into account to generate in silico approaches is the difference between visual assessment and automated methods. In [20], a comparison between visual assessment and Automated Digital Analysis (DIA) of Ki-67 is presented. That method demonstrates agreement between manual expert assessment-by eye-and DIA of Ki67 in breast cancer. However, the datasets for these studies are not publicly available, limiting their reproduction and comparison with similar approaches. A method to segment and characterize cells in carcinoma images stained with Ki-67 using K-means clustering and the watershed algorithm is proposed in [19]. The method calculates $75.1 \pm 6.7 \%$ positive nuclei compared with ImmunoRatio, $77.9 \pm 7.1 \%$, and manual, $71.4 \pm 5.5 \%$. However, this approach has not been tested on breast cancer images and the dataset is not publicly available. Furthermore, the aforementioned studies focus on Ki-67, which is a nuclear biomarker, rather than a cytoplasmic biomarker. In [21], an Automated Quantitative Analysis (AQUA) of biomarker expression detection with cytoplasmic or nuclear staining is described. This approach has been validated in tissue microarrays, as well as in whole tissue sections using images from ovarian and breast cancer [23]. However, AQUA employs multiplexed fluorescent stains to compartmentalize and measure expression of specific biomarkers and the image data are not publicly available. Bankhead et al. [22] describe an open-source solution for digital pathology and whole slide image analysis and employed annotation, segmentation, detection and classification using machine learning techniques. 
Advances have been made by our group in the classification of healthy tissues and organs using histological images, for example using Masson's trichrome and H\&E [24]. Image processing techniques, tissue and organ morphological information, image features-color and texture-clustering, supervised learning and deep learning to identify fundamental tissues [25-28] and organs [29-31] have been used in our approach.

In this paper, we present an in silico approach to classify and quantify IHC staining of the predominantly cytoplasmic marker, Wnt-1, in breast cancer. We used color deconvolution, K-means algorithm and Allred score [32] to recognize and quantify Wnt-1 levels. We propose that in silico methods such as this have a unique advantage of being able to reduce subjectivity and optimize visual scoring in greater detail.

This paper is structured as follows: the problem statement is presented in Section 4.1. The proposed approach to automatic classification and quantification of Wnt-1 expression is explained in detail in Section 4. The dataset, the experiments and the results are described in Section 2. In Section 3, the study is discussed. Finally, the main conclusions of this work are drawn in Section 5.

\section{Experiments and Results}

In this section, we present the complete process to evaluate the proposed approach. We show the results obtained in three subsections: (i) block-based Wnt-1 segmentation; (ii) block-based Wnt-1 classification; and (iii) Wnt-1 classification in a TMA tissue core image. Some measurements were calculated through subjective evaluation of a group by two experts of the research laboratory (R.K. and E.O.). The human is the best judge to evaluate the output of the segmentation algorithm, owing to the difficulty of obtaining ground truth for real images [33]. Many segmentation methods are assessed according to expert criteria [34]. Appealing to human intuition was convenient in our case, since our goal was to create a large dataset that can later be used to train our automated system. Taking each TMA tissue core image into account and the criteria defined using the Allred score, Wnt-1 expression was classified as follows: (i) proportion of positive cells based on five levels-1 $=(0 \%, 1 \%]$, $2=(1 \%, 10 \%], 3=(10 \%, 33 \%], 4=(33 \%, 66 \%]$, and $5=(66 \%, 100 \%]$, where the parentheses indicate an open interval that does not include its endpoints, and the square brackets indicate a closed interval that includes its endpoints; (ii) intensity score based on three levels- 1 is low, 2 is intermediate and 3 is strong (see Figure 1); and (iii) Allred score is the sum of the proportion of positive tumor cells and the intensity of immunostaining in those cells, giving a final score of 0 (negative) or between 2 and 8. On the other hand, F-score and subjective measures were used to assess the response of this work in the classification and quantification process. The ICC was used to estimate inter-rater reliability on quantitative data because it is highly flexible [35]. The ICC inter-rater agreement measures were interpreted following guidelines by Terry [36]: (i) Less than 0.50, poor; (ii) between 0.50 and 0.75 , moderate; (iii) between 0.75 and 0.90 , good; and (iv) between 0.90 and 1.00, excellent.

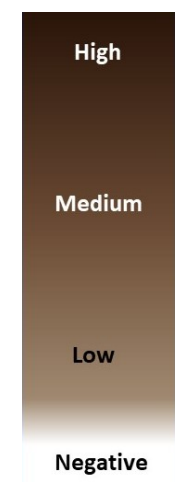

Figure 1. Labels and corresponding description for each one of the classes considered according to Wnt-1 intensity at pixel levels, four quarterlies are identified. 


\subsection{Evaluation of Block-Based Wnt-1 Segmentation}

A selected set of image blocks of different TMA tissue core images, Wnt-1 segmentation results and Wnt-1 classification according to Allred score is shown in Figure 2. Classification results are represented using watermark with distinctive colors as follows: (i) white represents [0-1] Allred score; (ii) yellow represents [2-3] Allred score; (iii) orange represents [4-6] Allred score; and (iv) red represents [7-8] Allred score.

Results of the automated Wnt-1 segmentation were evaluated by two experts and using a scale from 1 to 5 to represent poor, average, good, very good, and excellent. The Wnt-1 TMA staining was further reviewed by two pathologists (I.Z. and M.V.), whose analysis was compared to the final automated staining data. Figure 2 shows that Wnt-1 staining is absent from cell nuclei, consistent with $W n t-1$ being a cytoplasmic biomarker. Figure 3 contains a graphical representation of the mean of the evaluations of the ability of the approach to recognize Wnt-1 positive cells in the set of test block images.

a)

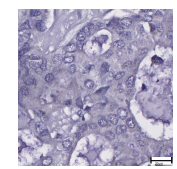

b)

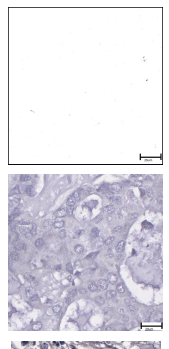

c)

d)

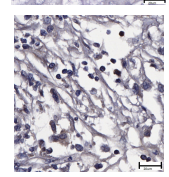

e)

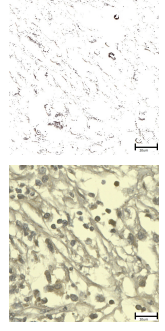

f)

g)

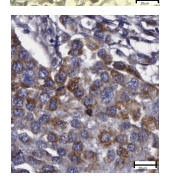

h)

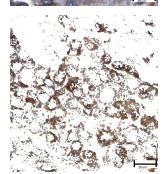

i)

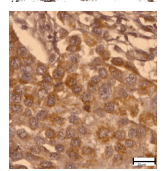

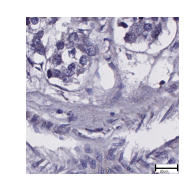
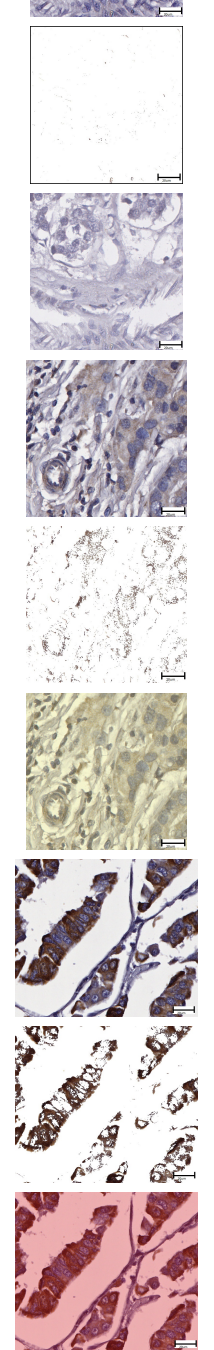
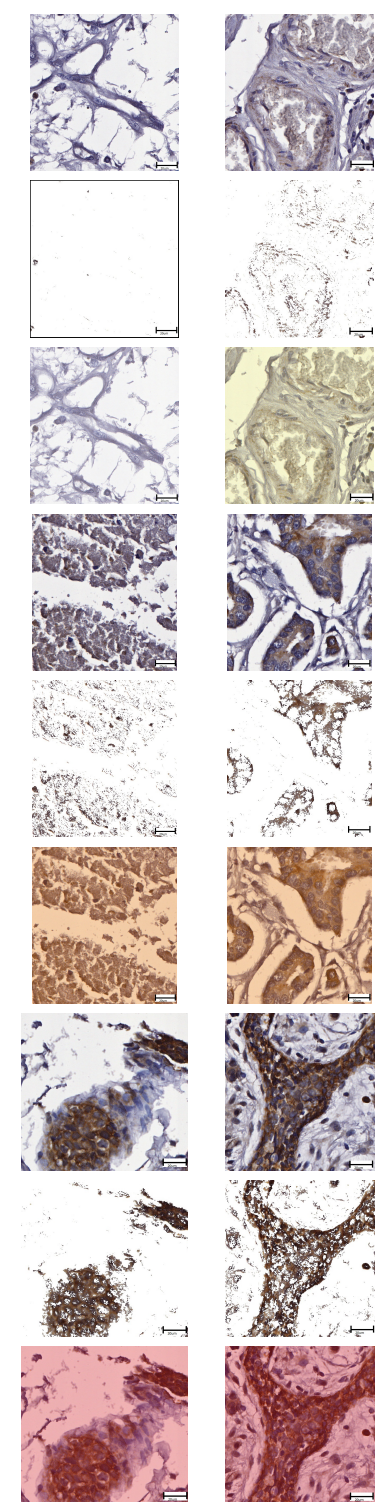
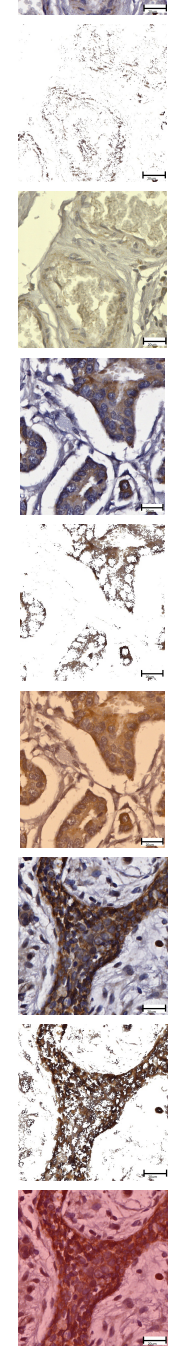

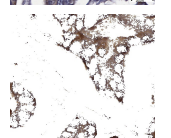

Figure 2. Selected Wnt-1 segmentation and classification results: $(\mathbf{a}, \mathbf{d}, \mathbf{g})$ original image blocks are presented; $(\mathbf{b}, \mathbf{e}, \mathbf{h})$ automatic segmentation using color convolution; and $(\mathbf{c}, \mathbf{f}, \mathbf{i})$ automatic Allred score classification, scale bar $=20 \mu \mathrm{m}$. 


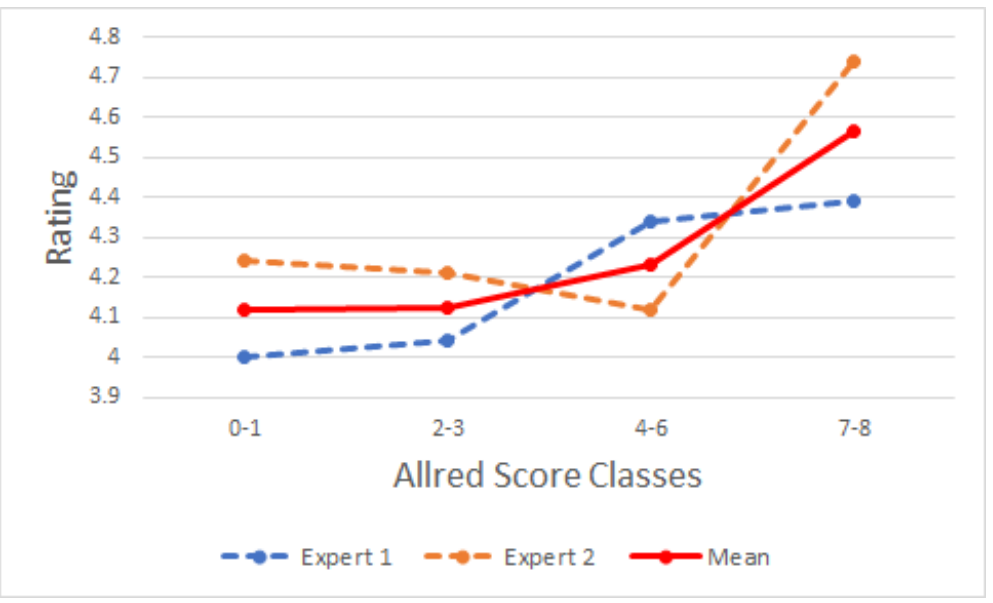

Figure 3. Results obtained from Wnt-1 positive segmentation. The y-axis is not displayed from the origin to improve visualization.

The ability of the proposed approach to recognize Wnt-1 was given an average score of 4.26 by the experts. The highest average was obtained for the [7-8] class and the lowest average was obtained for the [0-1] class. This lower score is due to the potential confusion between Wnt-1 and artifactual staining and/or debris and signal in the stroma that had not been removed. An advantage of the approach used is the segmentation of small Wnt-1 positive areas-sometimes imperceptible to the eye-and the possibility to evaluate the complete sample with pixel precision.

\subsection{Evaluation of Block-Based Wnt-1 Classification}

The classification results are shown in Figure 2. The confusion matrices [37] of automatic Wnt-1 classification, with each expert's evaluation, for four classes using Allred classification-[0-1], [2-3], [4-6] and [7-8] -are presented in Tables 1 and 2, respectively. Additionally, a graphical representation of F-score measures is illustrated in Figure 4. The results obtained were calculated using the set of block test images. The results obtained yield between 0.338 and 0.804 F-score using our approach. The highest F-score was obtained for the [7-8] class in both cases. The lowest F-scores were achieved for the [2-3] class in both cases, owing to its high similarity with the [4-6] class, taking into account the presence of non-tumor cells, apoptotic nuclei and debris. ICCs of $0.634,0.791,0.551$ and 0.630 were obtained for the classes [0-1], [2-3], [4-6] and [7-8], respectively.

Table 1. Confusion Matrix of Automatic Classification Based on Allred Score with Expert-1. The best F-score value is presented in bold.

\begin{tabular}{cccccc}
\hline & {$[\mathbf{0 - 1}]$} & {$[\mathbf{2 - 3}]$} & {$[4-6]$} & {$[7-8]$} & F-Score \\
\hline$[0-1]$ & 38 & 5 & 0 & 0 & 0.531 \\
{$[2-3]$} & 62 & 34 & 5 & 0 & 0.338 \\
{$[4-6]$} & 0 & 54 & 88 & 23 & 0.664 \\
{$[7-8]$} & 0 & 7 & 7 & 76 & $\mathbf{0 . 8 0 4}$ \\
\hline
\end{tabular}

Table 2. Confusion Matrix of Automatic Classification Based on Allred Score with Expert-2. The best F-score value is presented in bold.

\begin{tabular}{cccccc}
\hline & [0-1] & [2-3] & [4-6] & [7-8] & F-Score \\
\hline$[0-1]$ & 47 & 7 & 1 & 0 & 0.606 \\
{$[2-3]$} & 47 & 37 & 3 & 0 & 0.396 \\
{$[4-6]$} & 6 & 53 & 86 & 37 & 0.610 \\
{$[7-8]$} & 0 & 3 & 10 & 63 & $\mathbf{0 . 7 1 6}$ \\
\hline
\end{tabular}




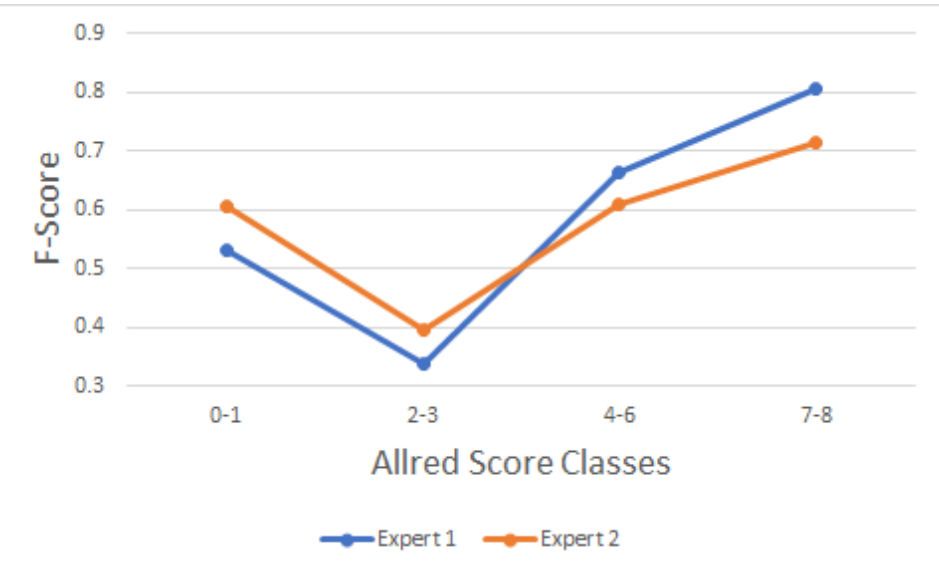

Figure 4. F-Score obtained with Wnt-1 automatic classification using Expert-1 and Expert-2's Allred score. [0-1] represents no affect, [2-3] represents small, [4-6] represents moderate, and [7-8] represents good. The y-axis is not displayed from the origin to improve visualization.

Analysis of the confusion matrices shows that high risk classes were correctly classified, while low risk classes presented some confusion with adjacent classes. This is due to the similarity between adjacent classes. Nevertheless, the results illustrate the extent to which the block-based automatic classification matches manual scoring.

\subsection{Evaluation of Wnt-1 Classification in a TMA Tissue Core Image}

Complete TMA results were obtained considering the TMA information, the result obtained (see Figure 5) and expert evaluation. We calculated the average ICC obtained as 0.752, showing a moderate degree of agreement between the evaluators and the automated classification.

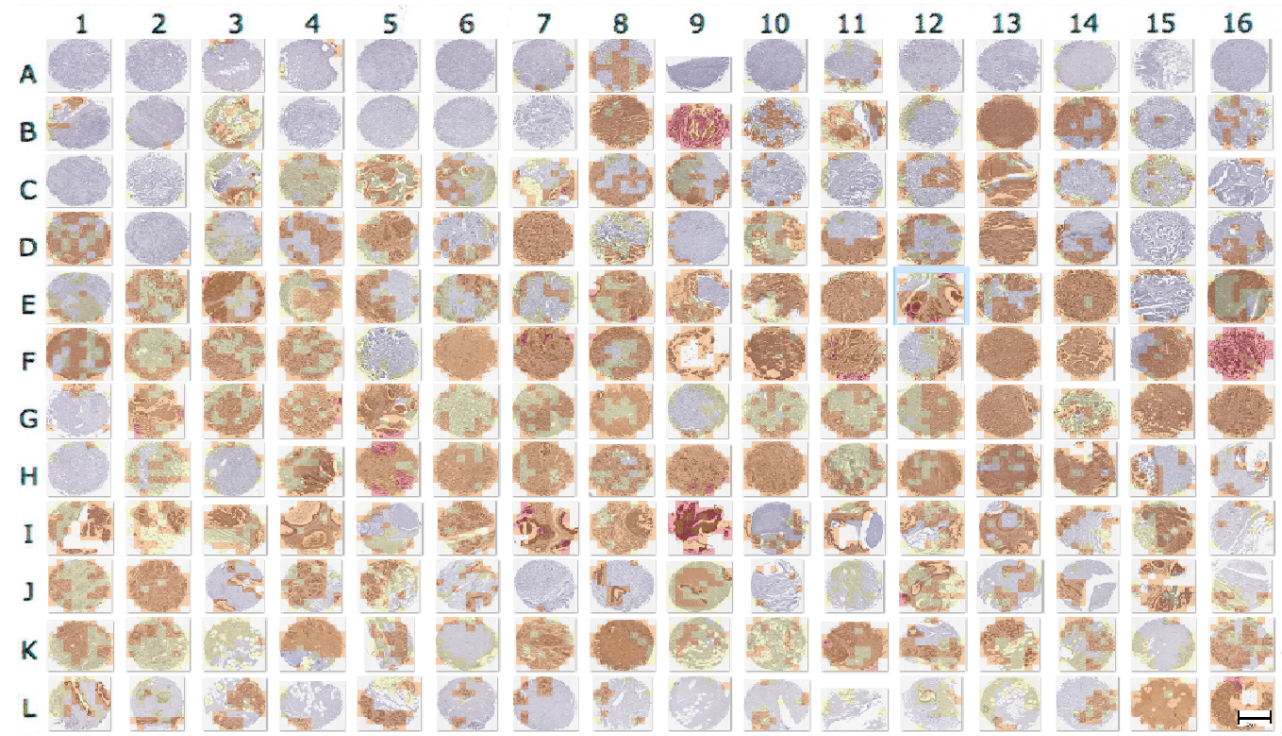

Figure 5. Results obtained for Wnt-1 positive classification in complete TMA tissue core images, scale bar $=20 \mu \mathrm{m}$.

\section{Discussion}

Color deconvolution is a robust and flexible method to identify and separate the DAB signal of the stain used. Color deconvolution and its variants have been used successfully in different histological 
and histopathological applications [38], showing advantages in determining staining densities, ratios and even for recognizing different structures.

The results obtained cannot be compared directly to other approaches in the literature because, although there are other solutions for automated biomarker identification, these use proprietary software [21] or are based on nuclear biomarkers [18,20]. Nonetheless, AQUA uses immunofluorescence intensity data to measure expression and, in some cases, is supported by additional information such as sub-cellular localization. AQUA has been used to measure several markers, including EGFR, ER, mTOR and PTEN. We compared the algorithm proposed in [18], called ImmunoRatio, with our method. Figure 6 illustrates the contrast among methods and shows that ImmunoRatio: (i) includes cell nuclei in biomarker identification; and (ii) separates particles trying to simulate cell nuclei in DAB areas.

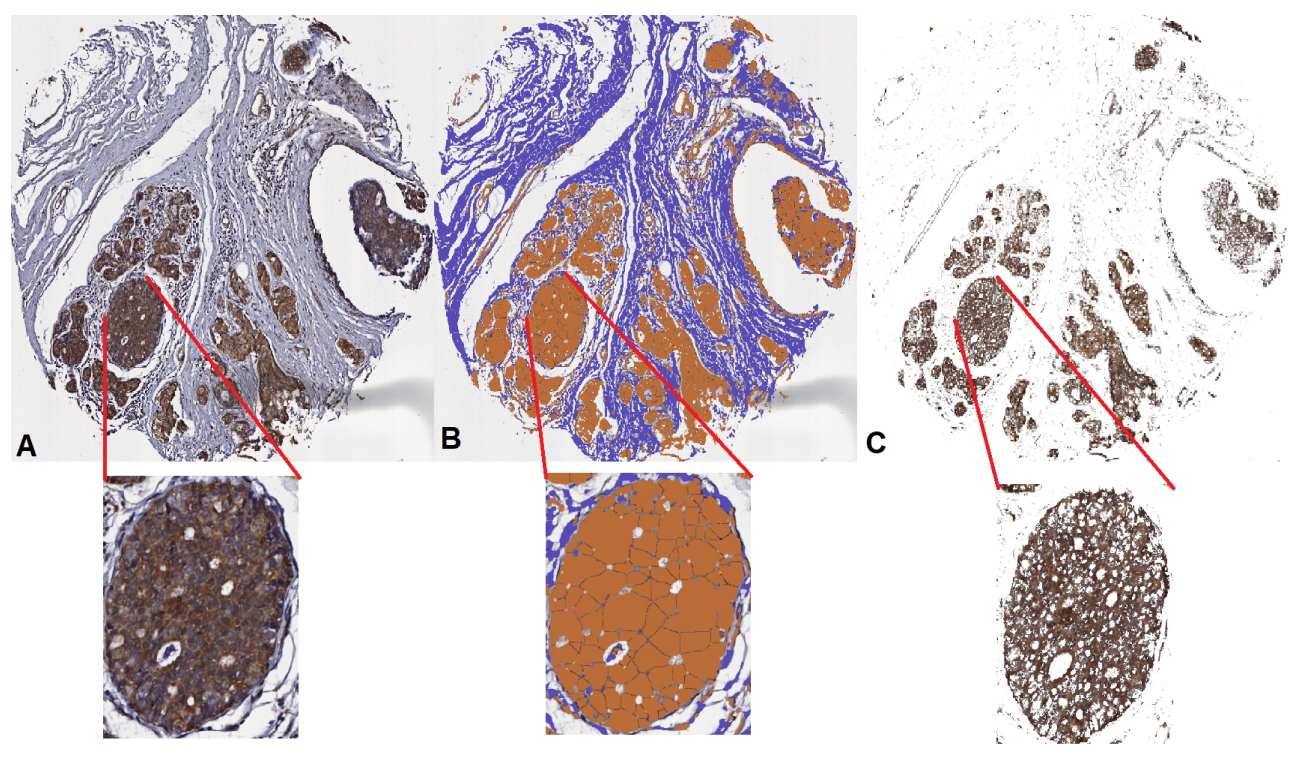

Figure 6. Comparison between immunoRatio and NUBIAWnt-11Ratio: (A) original TMA tissue core image; (B) ImmunoRatio results; and (C) NUBIAWnt-11Ratio results.

Our proposed method is based on dividing the image into image blocks, using color deconvolution and a K-means algorithm. Experimental evaluation has shown that our approach identified and quantified Wnt-1 levels in a similar way as an approach that would be used in the clinic.

\section{Materials and Method}

\subsection{Problem Statement}

The level of expression of Wnt-1 may be a biomarker for some breast cancers. However, Wnt-1 expression may vary in the same sample, according to the selected region of interest in the image (see Figure 7) or the level of Wnt-1 expression in the patient sample (see Figure 8). Color is the most important feature to analyze IHC in histopathology images [39]. One of the most relevant problems is that environmental factors and image acquisition devices can affect image quality and automated results. We used a TMA that was captured using an Aperio Digital Pathology Slide Scanner to achieve high quality images and reduce thermal drift and colour balance. There are also other types of variation in sectioning and staining processes and our approach increases robustness using color deconvolution and a clustering algorithm. Clinical researchers often use the Allred score to score samples manually. Allred score considers two aspects: (i) the proportion of cells that are positive over the evaluated area; and (ii) the intensity level of the positive staining. Pathologists normally examine larger areas or multiples sections to confirm their observations. The details of the complete process are presented in the following section. 
(a)

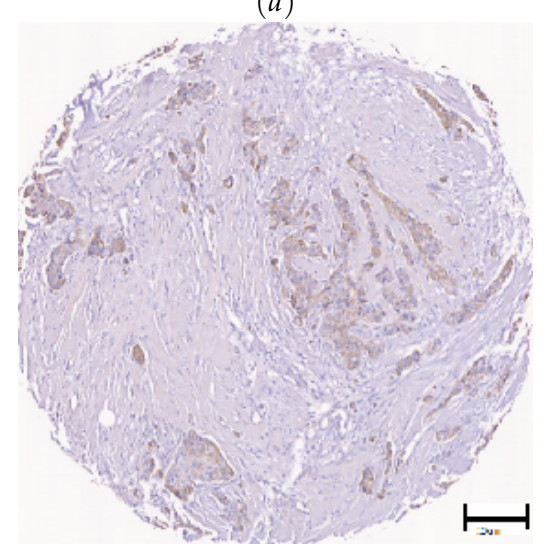

$(b)$

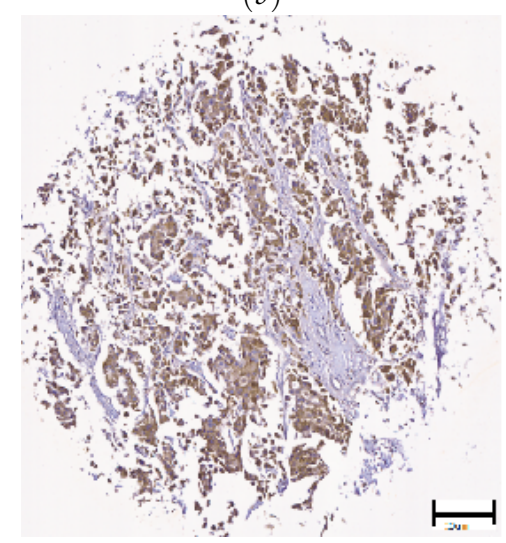

Figure 7. Illustration of stained cases of Wnt-1: (a) heterogeneously; and (b) homogeneously, scale bar $=20 \mu \mathrm{m}$.

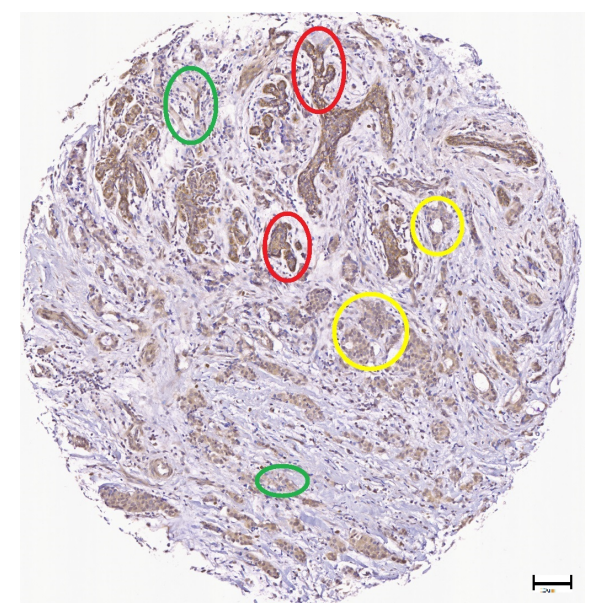

Figure 8. Illustration of Wnt-1 intensity level variation. The red circles correspond to high intensity level examples, the yellow circles correspond to medium level examples and the green circles correspond to low intensity level examples, scale bar $=20 \mu \mathrm{m}$.

Using Wnt-1 as an example, we propose a method for in silico classification and quantification of a cytoplasmic biomarker in breast cancer. A brief summary of the proposed process is given below and a detailed explanation is presented in the following subsections. Our method is composed of four steps: (i) a TMA tissue core image is divided into blocks; (ii) DAB areas are identified for each block using color deconvolution; (iii) DAB areas are classified using K-means algorithm by blocks; and (iv) each core image is classified using block-based classification. Figure 9 shows a general outline of our approach. (1) A set of TMA tissue core images are obtained from one or many TMAs. (2) Each TMA tissue core image is processed. (3) Blocks of $m \times n$ pixels are obtained from the input image. In this study, 64 blocks by image were obtained. (4) After evaluating different methods to identify DAB, color deconvolution is used to separate: (4.1) Hematoxylin; (4.2) Eosin; and (4.3) DAB. (5) Pixels that represent tissues are identified using (4.1) and (4.2). A pixel position is represented by a three-element feature vector Red, Gree and Blue (RGB) representing the amount of each colour in that position contains. (6) The input for the K-means algorithm is composed by the set of RGB vectors for the $\mathrm{DAB}$ image and the $K$ parameter, representing the number of clusters to obtain, which has been set to four groups: (6.1) high Wnt-1 positive intensity levels; (6.2) medium Wnt-1 positive intensity levels; (6.3) low Wnt-1 positive intensity levels; and (6.4) light regions (background and spaces between tissues). The black regions correspond to segmented areas. (7) Tissues and DAB proportion measures 
are calculated. (8) Blocks are classified by Allred score and then segmentation of the entire image is formed by stitching classified blocks. (9) A classified TMA tissue core image is obtained. The details of the complete process are presented in this section.

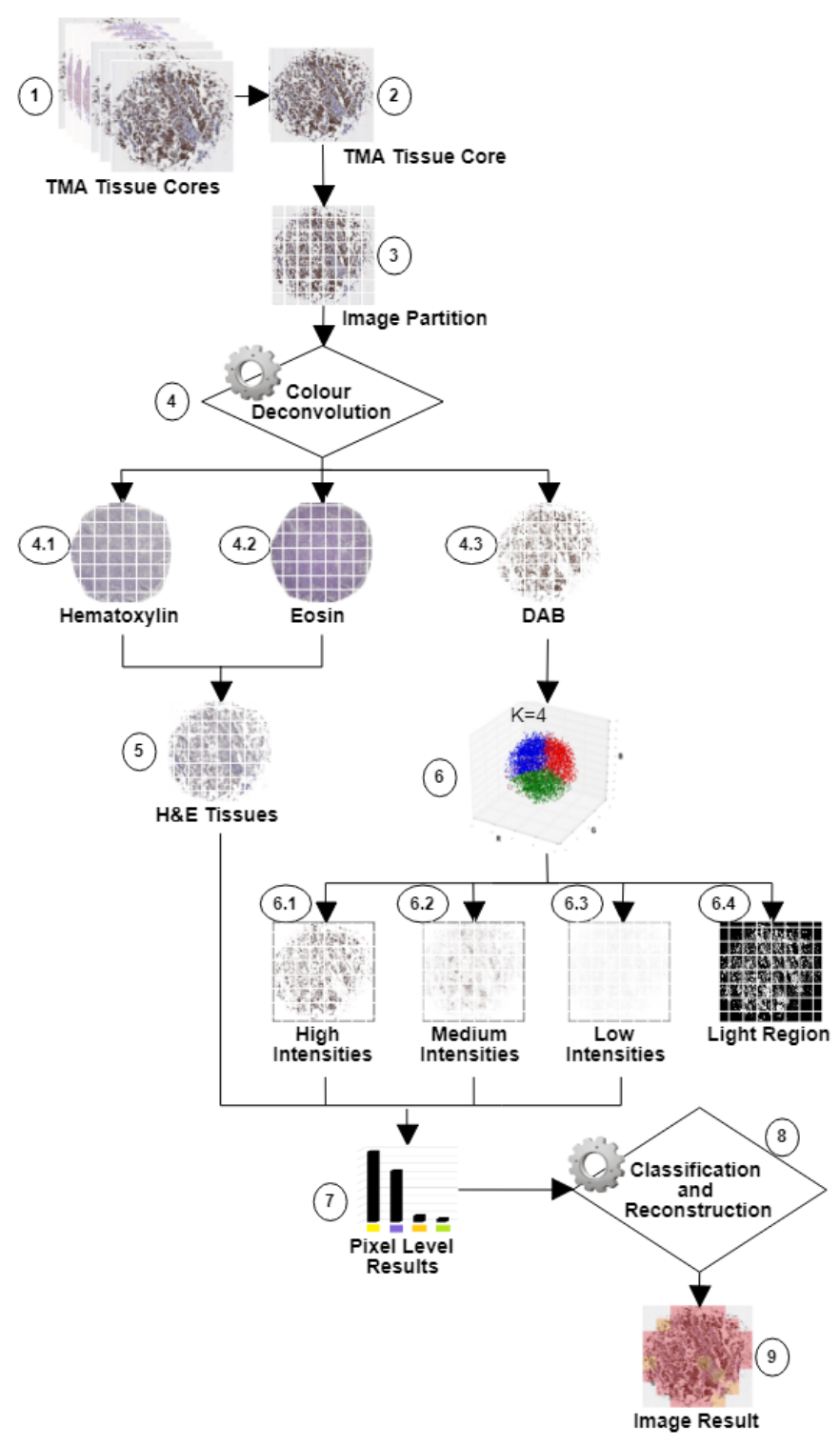

Figure 9. Proposed approach for automated segmentation and quantification of Wnt-1: (1) TMA tissue core images with DAB (brown), hematoxylin (blue) and eosin (magenta). (2) TMA tissue core image. (3) 64 image blocks. (4) Color Deconvolution process. Color Deconvolution results: (4.1) hematoxylin, (4.2) eosin and (4.3) DAB of the original image. (5) Tissues without DAB. (6) Clustering process with K-means algorithm using initial labels $k=4$. (6.1) Wnt-1 high intensities. (6.2) Wnt-1 medium intensities. (6.3) Wnt-1 low intensities. (6.4) Light regions. (7) Quantification results at pixel level. (8) Classification and reconstruction using Allred score. (9) Image result. In the resulting images, the black or color regions correspond to segmented areas.

\subsection{Experimental Setup}

Tissue specimen sample cores in the breast TMA were immunostained for the Wnt-1 protein. The TMA was composed of 192 tissue cores, we used one image per tissue core, and 12, 288 image blocks were used for validation $(70 \%)$ and testing $(30 \%)$. The images were acquired with variable pixels of resolution according to each tissue core, between $3968 \times 4970$ and $5400 \times 5500$, and the images were 
stored in JPG format. To avoid introducing additional margins of error, the images were not modified further. Block sizes varied according to the pixel resolution of each TMA core image, minimizing differences between blocks. The datasets belonging to image blocks obtained from different samples and patients were acquired using a $20 \times$ objective. Two scientists familiar with immunohistochemical analysis of TMAs reviewed the TMA tissue core images blindly and graded the cytoplasmic staining for Wnt-1 intensity and percentage of positive cells, according to the Allred scoring method. An Aperio Digital Pathology Slide Scanner with eyepieces with a magnification factor of $10 \times$ and a field of view of 20 obtaining 200 end magnification for a $20 \times$ objective was used. We have made the datasets publicly available at: https:/ / vicomtech.box.com/v/Wnt1Dataset. Algorithms were implemented in Python, using the OpenCV library for computer vision [40], on a computer with 4 cores, 8 GB memory and a NVIDIA Titan X Pascal GPU.

\subsection{Partitioning TMA Tissue Cores into Blocks}

Our approach is to identify Wnt-1-positive areas using a block-based strategy. A block is the analysis unit to identify, classify and quantify Wnt-1-positive areas in an image. A block is a fixed non-overlapping $m \times n$ partition of a TMA tissue core image. The block size depends on the original image size; 64 blocks are obtained per image. The number of blocks was decided heuristically taking into account that, if the block size is too small or too large, high variations may hinder its analysis.

\subsection{Block-Based Wnt-1 Segmentation}

Color information is a discriminant feature for IHC staining analysis. We use the color deconvolution strategy proposed in [41]. This method is based on orthonormal transformation of the original RGB image of samples stained with H\&E and DAB at different staining levels. This method is composed of two steps: (i) color representation; and (ii) color deconvolution. The method proposed in [41] provides a robust and flexible method for objective IHC analysis of samples; it provides the possibility to determine staining densities even in areas where multiple stains are co-localized, making it possible not only to determine surface area and overall absorption in areas with a specific colour, but also to determine densities and ratios of densities of stains in each area (see Figure 10).

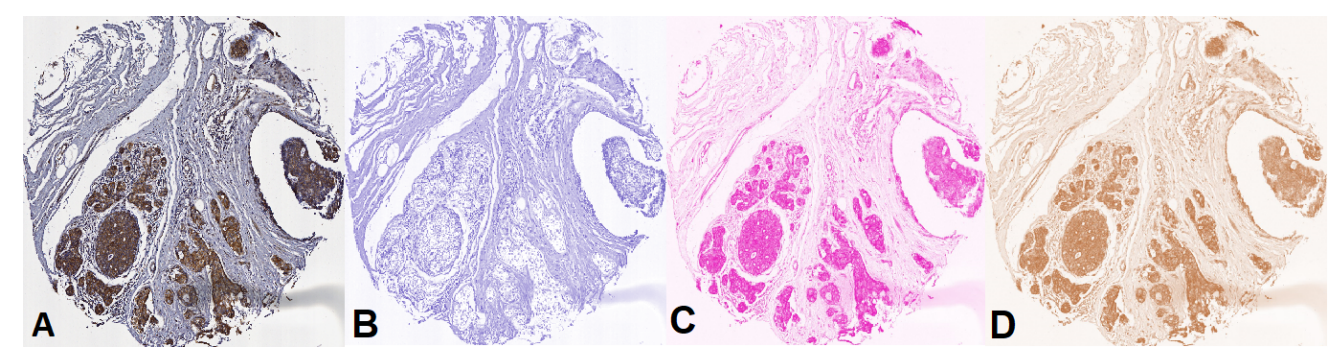

Figure 10. Colour deconvolution in histopathological images: (A) original TMA tissue core image; and (B-D) color deconvolution results separating the contribution of hematoxylin (B), eosin (C) and DAB (D) to the original image. Magnification $20 \times$.

\subsection{Block-Based Wnt-1 Classification}

In this proposal, we classify blocks into four classes at pixel level—high intensity, medium intensity, low intensity and light regions-using the K-means algorithm with $k=4$. The K-mean's inputs are the initial centers-during the first attempt, we used the user-supplied labels instead of computing them from the initial centers-and the RGB values. Initial centers were established heuristically taking into account the expert's evaluation for intensity level of Wnt-1 positive.

A laboratory protocol and an image capture protocol were defined to have an image dataset with similar characteristics and to reduce errors in the automatic evaluation. However, thermal drift and color balance affect the analysis introducing small variations in the intensities of the colors and lighting 
and other variations may be introduced during the tissue staining process. This situation implies that the proposed method has to be robust to small variations in color balance and thermal drift that may occur. Invariance is granted by the K-means algorithm and the protocols.

Let $I: \mathbb{I} \times \mathbb{I} \rightarrow \mathbb{R}^{3}$ be a block of size $m \times n$ pixels in $R G B$ color space; $H_{k}(t)$ is a cluster represented by a set of vectors in $\mathbb{R}^{3}$ in the $t$ th iteration; and $C_{k}(t) \in \mathbb{R}^{3}$ be a centroid $K$ of the cluster $H_{k}(t)$. We use RGB values since they contain relevant information about IHC and H\&E. The initial parameters of the K-means algorithm are set: $t=0, C_{1}(0)=\{64,32,21\}, C_{2}(0)=\{105,51,27\}, C_{3}(0)=\{124,87,45\}$, $C_{4}(0)=\{255,255,255\}$. We write K-means in two steps: (1) assignment step where each pixel $I_{i j}$ is assigned to the cluster $H_{k}$ which centroid $C_{k}$ is the closest in the Euclidean way:

$$
I_{i j} \in H_{k}(t) \text { if } k=\underset{k \in\{1,2,3,4\}}{\arg \min }\left\|I_{i j}-C_{k}(t)\right\| ;
$$

and (2) an update step where each centroid $C_{k}$ is updated based on the observations that belong to its cluster $H_{k}$ :

$$
C_{k}(t+1)=\frac{1}{\left|H_{k}(t)\right|} \sum_{i=1}^{M} \sum_{j=1}^{N} a_{i j} . I_{i j}
$$

where $a_{i j}$ is $I_{i j}$ if $I_{i j} \in H_{k}(t)$ and 255 in other case. These two steps are carried out iteratively until convergence. Let $O=\left\{O_{1}, O_{2}, O_{3}, O_{4}\right\}$ be a image in $R G B$ such that:

$$
\left(O_{k}\right)_{i j}= \begin{cases}I_{i j} & I_{i j} \in H_{k}(t) \\ \{255,255,255\} & \text { else, }\end{cases}
$$

where a value of $k$ represents a different class in $I$, such that $k=1$ corresponds to high DAB intensities pixel positions (associated with cytoplasm), $k=2$ corresponds to medium DAB intensities, $k=3$ corresponds to low DAB intensities, and $k=4$ corresponds to light regions. Thus, we obtain DAB levels in an image block with the $R G B$ values of $I$.

\subsection{Wnt-1 Classification in a TMA Tissue Core Image}

Wnt-1 classification in a TMA tissue core image using the block-based Wnt-1 classification (see Figure 9, Steps (8) and (9)) is defined as:

Let $I: \mathbb{I} \times \mathbb{I} \rightarrow \mathbb{R}^{3}$ be a TMA tissue core image in $R G B$ colour space; $I_{s}=\left\{I_{0}, I_{1}, \ldots, I_{K}\right\}$ be a TMA or a set of TMAs; $B$ be a matrix of blocks in which each $B_{i j}$ represents the $j$ th block of the image $i$; $M\left(B_{i j}\right)$ be the block-based $W n t-1$ classification method; $W c_{I}$ be a $m \times m$ matrix of labels where $m=8$. Then, Wnt-1 classification of a TMA tissue core image using block-based recognition is:

$$
W c_{I}=\left(\begin{array}{cccc}
M\left(B_{11}\right) & M\left(B_{12}\right) & \cdots & M\left(B_{1 m}\right) \\
M\left(B_{21}\right) & M\left(B_{22}\right) & \cdots & M\left(B_{2 m}\right) \\
\vdots & \vdots & \cdots & \vdots \\
M\left(B_{m 1}\right) & M\left(B_{m 2}\right) & \cdots & M\left(B_{m m}\right)
\end{array}\right)
$$

\section{Conclusions}

In this paper, we present an in silico approach that allows the classification and quantification of a cytoplasmic protein in breast cancer histopathological images with an average F-score and accuracy greater than $0.58 \%$ and $97 \%$ according to the class of risk to identified, being more precise for the high risk classes ([7-8] Allred Score).

High variability between expert's evaluations are due to the subjective criteria used-proportion and intensity. The misclassified classes resulted from additional features, including the proportion of tumor cell cells and signal from stromal cells and apoptotic cells. However, average ICC was improved with the proposed approach. 
Using the recognized Wnt-1 positivity from a block of size $m \times n$, we were able to classify it according to Allred score. Taking into account these results, it is possible to classify TMA tissue core images by extracting the appropriate segmentation with the selection of the proper classifier. Color deconvolution is a robust and flexible method that determines density and ratios of densities of stains in each area. In addition, the proposed in silico approach is faster than the traditional manual approach.

Using markers such as Wnt-1 may in future identify breast cancer patients with a high risk of tumor recurrence and/or progression to metastasis, who may then benefit from further intensive therapy after a surgery [6].

We have created and made publicly available a dataset consisting of 12, 288 image blocks-192 TMA tissue cores images- that can be used to validate the results obtained in our work or to improve upon the proposed method.

In the future, we will extend this proposal through the following five lines of investigation: (i) develop an approach that excludes stromal cells and return a classification by tissue core; (ii) integrate our approach with other cytoplasmic and nuclear biomarkers (e.g., Ki-67, ER, PR, and Sox2); (iii) evaluate ROIs with different shapes; (iv) explore new classification techniques, such as deep learning algorithms; and (v) compare our proposal with other approaches in the literature.

Author Contributions: Conceptualization, C.M., M.d.M.V., I.Z. and A.B.; Data curation, C.M., E.O.E., M.d.M.V. and R.M.K.; Formal analysis, C.M.; Funding acquisition, C.M. and M.d.M.V.; Investigation, C.M.; Methodology, C.M.; Project administration, C.M. and M.d.M.V.; Resources, M.d.M.V. and A.B.; Software, C.M.; Supervision, M.d.M.V. and A.B.; Validation, C.M., E.O.E., I.Z., M.d.M.V. and R.M.K.; Visualization, C.M.; Writing-original draft, C.M.; and Writing—review and editing, C.M., E.O.E., M.d.M.V., R.M.K. and A.B..

Funding: This work was funded by Basque Government by means of the RIS3 Program. In addition, the authors were funded by the Department of Industry, Tourism and Trade (Elkartek) and Department of Innovation Technology of the Government of the Autonomous Community of the Basque Country and Centre of Excellence Severo Ochoa (2017-2021) (EO, MV, and RK), Basque Country Department of Education PRE_2014_1_293 (EO), the Spanish Ministry of Science and Innovation (MICINN SAF2017-84934-R (RK) and SAF2017-84092-R (MV)) and ACAMBI (MV). CM holds a grant from the EI and from the European Union's Horizon 2020 research and innovation programme under the Marie Slodowska-Curie grant agreement No. 713654.

Acknowledgments: We gratefully acknowledge the support of NVIDIA Corporation with the donation of the Titan Xp GPU used for this research.

Conflicts of Interest: The authors declare no conflict of interest.

\section{Abbreviations}

The following abbreviations are used in this manuscript:

DAB Diaminobenzidine

H\&E Hematoxylin and Eosin

TMA Tissue Microarray

ICC Intraclass Correlation

IHC Immunohistochemistry

MMTV Mouse Mammary Tumour Virus

CSC Cancer Stem Cell

ER Estrogen Receptor

ER+ Estrogen Receptor-positive

PR Progesterone Receptor

DIA Automated Digital Analysis

AQUA Automated Quantitative Analysis

RGB Red, Green and Blue 


\section{References}

1. Torre, L.A.; Bray, F.; Siegel, R.L.; Ferlay, J.; Lortet-Tieulent, J.; Jemal, A. Global cancer statistics, 2012. CA A Cancer J. Clin. 2015, 65, 87-108. doi:10.3322/caac.21262. [CrossRef] [PubMed]

2. American Cancer Society medical, How Common Is Breast Cancer? 2018. Available online: https://www. cancer.org/cancer/breast-cancer/about/how-common-is-breast-cancer.html (accessed on 15 January 2018).

3. Cencer Research UK, Cancer Incidence for Common Cancers, 2016. Available online: http:/ / www.cancerresearchuk. org/health-professional/cancer-statistics/incidence/common-cancers-compared\#heading-Zero (accessed on 15 January 2018).

4. American. Cancer Society and International Agency of Research Cancer, in Women, Breast Cancer Is the Most Common Cancer and the \#1 Cause of Cancer Death, 2012. Available online: http:/ / canceratlas.cancer. org/the-burden/breast-cancer/ (accessed on 10 October 2018).

5. Zhan, T.; Rindtorff, N.; Boutros, M. Wnt-1 signaling in cancer. Oncogene 2017, 36, 1461-1473. [CrossRef] [PubMed]

6. Piva, M.; Domenici, G.; Iriondo, O.; Rábano, M.; Simões, B.; Comaills, V.; Barredo, I.; López-Ruiz, J.; Zabalza, I.; Kypta, R.; et al. Sox2 promotes tamoxifen resistance in breast cancer cells. EMBO Mol. Med. 2014, 6, 66-79. [CrossRef] [PubMed]

7. Varmus, R.N.H.E. Many tumors induced by the mouse mammary tumor virus contain a provirus integrated in the same region of the host genome. Cell 1982, 31, 99-109.

8. Vaillant, F.; Asselin-Labat, M.L.; Shackleton, M.; Forrest, N.C.; Lindeman, G.J.; Visvader, J.E. The Mammary Progenitor Marker CD61/I3 Integrin Identifies Cancer Stem Cells in Mouse Models of Mammary Tumorigenesis. Cancer Res. 2008, 68, 7711-7717. [CrossRef] [PubMed]

9. Jang, G.B.; Kim, J.Y.; Cho, S.D.; Park, K.S.; Jung, J.Y.; Lee, H.Y.; Hong, I.S.; Nam, J. Blockade of Wnt-1/ $\beta$-catenin signaling suppresses breast cancer metastasis by inhibiting CSC-like phenotype. Sci. Rep. 2015, 5, 12465. [CrossRef] [PubMed]

10. Choi, E.J.; Yun, J.A.; Jeon, E.K.; Won, H.S.; Ko, Y.H.; Kim, S.Y. Prognostic significance of RSPO1, WNT1, P16, WT1, and SDC1 expressions in invasive ductal carcinoma of the breast. World J. Surg. Oncol. 2013, 11, 314. [CrossRef]

11. Jiang, Q.; He, M.; Ma, M.; Wu, H.; Yu, Z.; Guan, S.; Jiang, L.; Wang, Y.; Zheng, D.; Jin, F.; et al. MicroRNA-148a inhibits breast cancer migration and invasion by directly targeting WNT-1. Oncol. Rep. 2016, 35, 1425-1432. [CrossRef]

12. Halifu, Y.; Liang, J.; Zeng, X.; Ding, Y.; Zhang, X.; Jin, T.; Yakeya, B.; Abudu, D.; Zhou, Y.; Liu, X.; et al. Wnt-11 and SFRP1 as potential prognostic factors and therapeutic targets in cutaneous squamous cell carcinoma. Genet. Mol. Res. 2016, 15, 1-7. [CrossRef]

13. Xu, X.; Sun, P.; Li, J.; Jheon, S.; Lee, C.; Chung, J. Aberrant Wnt-11/I-Catenin Expression is an Independent Poor Prognostic Marker of Non-small Cell Lung Cancer after Surgery. J. Thorac. Oncol. 2011, 6, 716-724. [CrossRef]

14. Lee, H.H.; Uen, Y.H.; Tian, Y.F.; Sun, C.S.; Sheu, M.J.; Kuo, H.T.; Koay, L.B.; Lin, C.Y.; Tzeng, C.C.; Cheng, C.J.; et al. Wnt-1 Protein as a Prognostic Biomarker for Hepatitis B-Related and Hepatitis C-Related Hepatocellular Carcinoma after Surgery. Cancer Epidemiol. Prev. Biomark. 2009, 18, 1562-1569. [CrossRef] [PubMed]

15. Henriksen, K.L.; Rasmussen, B.B.; Lykkesfeldt, A.E.; Møller, S.; Ejlertsen, B.; Mouridsen, H.T. Semi-quantitative scoring of potentially predictive markers for endocrine treatment of breast cancer: A comparison between whole sections and tissue microarrays. J. Clin. Pathol. 2007, 60, 397-404. [CrossRef] [PubMed]

16. Izet, M. E-Learning as New Method of Medical Education. Acta Inform. Med. 2008, 16, 102-117.

17. Hernández, A.I.; Porta, S.M.; Miralles, M.; García, B.F.; Bolúmar, F. La cuantificación de la variabilidad en las observaciones clínicas. Med. Clin. 1990, 424-429.

18. Tuominen, V.J.; Ruotoistenmäki, S.; Viitanen, A.; Jumppanen, M.; Isola, J. ImmunoRatio: A publicly available web application for quantitative image analysis of estrogen receptor (ER), progesterone receptor (PR), and Ki-67. Breast Cancer Res. 2010, 12, R56. [CrossRef] [PubMed] 
19. Shi, P.; Zhong, J.; Hong, J.; Huang, R.; Wang, K.; Chen, Y. Automated Ki-67 Quantification of Immunohistochemical Staining Image of Human Nasopharyngeal Carcinoma Xenografts. Sci. Rep. 2016, 6, 32127. [CrossRef] [PubMed]

20. Zhong, F.; Bi, R.; Yu, B.; Yang, F.; Yang, W.; Shui, R. A Comparison of Visual Assessment and Automated Digital Image Analysis of Ki67 Labeling Index in Breast Cancer. PLoS ONE 2016, 11, e0150505. [CrossRef]

21. Weberpals, J.I.; Amin, M.S.; Chen, B.E.; Tu, D.; Spaans, J.N.; Squire, J.A.; Eisenhauer, E.A.; Virk, S.; Ma, D.; Duciaume, M.; et al. First application of the Automated QUantitative Analysis (AQUA) technique to quantify PTEN protein expression in ovarian cancer: A correlative study of NCIC CTG OV.16. Gynecol. Oncol. 2016, 486-493, doi:10.1016/J.Ygyno.2016.01.015. [CrossRef]

22. Bankhead, P.; Loughrey, M.B.; Fernández, J.A.; Dombrowski, Y.; McArt, D.G.; Dunne, P.D.; McQuaid, S.; Gray, R.T.; Murray, L.J.; Coleman, H.G.; et al. QuPath: Open source software for digital pathology image analysis. Sci. Rep. 2017, 7, 16878. [CrossRef]

23. Gustavson, M.D.; Bourke-Martin, B.; Reilly, D.; Cregger, M.; Williams, C.; Mayotte, J.; Zerkowski, M.; Tedeschi, G.; Pinard, R.; Christiansen, J. Standardization of HER2 immunohistochemistry in breast cancer by automated quantitative analysis. Arch. Pathol. Lab. Med. 2009, 133, 1413-1419.

24. Fischer, A.; Jacobson, K.; Rose, J.; Zeller, R. Hematoxylin and Eosin Staining of Tissue and Cell Sections. Cold Spring Harb. Protoc. 2008. [CrossRef] [PubMed]

25. Mazo, C.; Trujillo, M.; Salazar, L. An Automatic Segmentation Approach of Epithelial Cells Nuclei. In Progress in Pattern Recognition, Image Analysis, Computer Vision, and Applications; Alvarez, L., Mejail, M., Gomez, L., Jacobo, J., Eds.; Springer Berlin/Heidelberg, Germany, 2012; Volume 7441, pp. 567-574.

26. Mazo, C.; Trujillo, M.; Salazar, L. Automatic Classification of Coating Epithelial Tissue. In Progress in Pattern Recognition, Image Analysis, Computer Vision, and Applications; Bayro-Corrochano, E.,Hancock, E., Eds.; Springer: Berlin, Germany, 2014; Volume 8827, pp. 311-318.

27. Mazo, C.; Trujillo, M.; Salazar, L. Identifying Loose Connective and Muscle Tissues on Histology Images. In Progress in Pattern Recognition, Image Analysis, Computer Vision, and Applications; Ruiz-Shulcloper, J., Sanniti di Baja, G., Eds.; Springer: Berlin/Heidelberg, Germany, 2013; Volume 8259, pp. 174-180.

28. Mazo, C.; Bernal, J.; Trujillo, M.; Alegre, E. Transfer learning for classification of cardiovascular tissues in histological images. Comput. Methods Programs Biomed. 2018, 165, 69-76. [CrossRef]

29. Mazo, C.; Trujillo, M.; Alegre, E.; Salazar, L. Automatic recognition of fundamental tissues on histology images of the human cardiovascular system. Micron 2016, 89, 1-8. [CrossRef] [PubMed]

30. Mazo, C.; Alegre, E.; Trujillo, M.P.; González-Castro, V. Tissues Classification of the Cardiovascular System Using Texture Descriptors. In Proceedings of the 21st Annual Conference onMedical Image Understanding and Analysis-MIUA 2017, Edinburgh, UK, 11-13 July 2017; pp. 123-132.

31. Trujillo, C.M.E.A.M. Classification of cardiovascular tissues using LBP based descriptors and a cascade SVM. Comput. Methods Programs Biomed. 2017, 147, 1-10.

32. Harvey, J.M.; Clark, G.M.; Osborne, C.K.; Allred, D.C. Estrogen Receptor Status by Immunohistochemistry Is Superior to the Ligand-Binding Assay for Predicting Response to Adjuvant Endocrine Therapy in Breast Cancer. J. Clin. Oncol. 1999, 17, 1474-1474. [CrossRef] [PubMed]

33. Heath, M.; Sarkar, S.; Sanocki, T.; Bowyer, K. Comparison of Edge Detectors. Comput. Vis. Image Underst. 1998, 69, 38-54. [CrossRef]

34. Unnikrishnan, R.; Pantofaru, C.; Hebert, M. Toward Objective Evaluation of Image Segmentation Algorithms. Ieee Trans. Pattern Anal. Mach. Intell. 2007, 29, 929-944. [CrossRef]

35. Koch, G.G. Intraclass correlation coefficient. In Encyclopedia of Statistical Sciences; Kotz Samuel, J.N.L., Ed.; John Wiley Sons: Hoboken, NJ, USA, 1982; Volume 4, pp. 213-217.

36. Koo, T.; Li, M.Y. A Guideline of Selecting and Reporting Intraclass Correlation Coefficients for Reliability Research. J. Chiropr. Med. 2016, 15, 155-163. [CrossRef]

37. Fawcett, T. An introduction to ROC analysis. Pattern Recognit. Lett. 2006, 27, 861-874. [CrossRef]

38. Onder, D.; Zengin, S.; Sarioglu, S. A review on color normalization and color deconvolution methods in histopathology. Appl. Immunohistochem. Mol. Morphol. 2014, 22, 713-719. [CrossRef]

39. Shu, J.; Dolman, G.E.; Duan, J.; Qiu, G.; Ilyas, M. Statistical colour models: An automated digital image analysis method for quantification of histological biomarkers. Biomed. Eng. Online 2016, 15, 46. [CrossRef] [PubMed] 
40. Itseez. Open Source Computer Vision Library. Available online: https://github.com/itseez/opencv (accessed on 15 Demceber 2018).

41. Ruifrok, A.C.; Johnston, D.A. Quantification of histochemical staining by color deconvolution. Anal. Quant. Cytol. Histol. 2001, 23, 291-299. [PubMed]

(C) 2018 by the authors. Licensee MDPI, Basel, Switzerland. This article is an open access article distributed under the terms and conditions of the Creative Commons Attribution (CC BY) license (http:/ / creativecommons.org/licenses/by/4.0/). 